\title{
A critical view on temperature modelling for application in weather derivatives markets
}

\author{
Jūratė Šaltytė Benth \& Fred Espen Benth
}

August 12, 2010

\begin{abstract}
In this paper we present a stochastic model for daily average temperature. The model contains seasonality, a low-order autoregressive component and a variance describing the heteroskedastic residuals. The model is estimated on daily average temperature records from Stockholm (Sweden). By comparing the porposed model with the popular model of Campbell and Diebold (2005), we point out some important issues to be adressed when modelling the temperature for application in weather derivatives market.
\end{abstract}

Keywords: temperature, time series model, weather derivatives, seasonality, GARCH 


\section{Introduction}

In recent years there has been a growing interest in modelling the dynamics of surface air temperature with application in pricing weather derivatives. We follow up this stream of research with a critical discussion on model building and estimation, contrasting two stochastic models proposed by Campbell and Diebold [14] and Benth and Šaltytė Benth [5]. Both models are based on a seasonal autoregressive (AR) process, but with significant differences in structure which influences their applicability in relation to temperature derivatives.

At the Chicago Mercentile Exchange (CME) there is an organized trade in weather futures and options. In particular, the CME offers trade in futures contracts written on temperature indices measured at various locations world wide, providing financial instruments to hedge weather risk exposure. The locations are major cities in the US, Canada, Europe and Asia. The temperature indices measure the daily cumulative average temperature (CAT), the cumulative heating-degree days (HDD) or the cumulative cooling-degree days (CDD). The reference temperature is taken as the average of the daily minimum and maximum temperature, wich we further refer to as temperature.

More specifically, if we denote the temperature on day $t$ by $Z(t)$, then the CAT index over a measurement period $\left[T_{1}, T_{2}\right]$ is defined as

$$
\operatorname{CAT}\left(T_{1}, T_{2}\right)=\sum_{t=T_{1}}^{T_{2}} Z(t),
$$

where the measurement period is typically a given month or season. At CME, CAT futures are traded on European cities for measurement periods 
in warm season. The HDD index measures the demand for heating in the cold period of the year, and is defined as the cumulative amount of average temperatures below a threshold over a measurement period. That is, one aggregates $\max (c-Z(t), 0)$, where the threshold $c$ is $65^{\circ} \mathrm{F}$ or $18^{\circ} \mathrm{C}$. The CDD index analogously aggregates $\max (Z(t)-c, 0)$ and measures the demand for air-conditioning cooling. The CDD and HDD futures are traded for US cities.

The temperature futures contracts are financially settled proportionally to the underlying index at the end of the measurement period. To assess the risk in trading such contracts and to be able to settle reasonable futures prices, one needs precise models for the temperature dynamics. A model should incorporate such properties as possible trend due to global warming and/or urbanisation, seasonal component describing periodic temperature variations related to cold and warm seasons, AR properties for temperature changes, and seasonal variations in residuals. In this study, we follow up the analysis from papers Benth and Šaltytė Benth [5], Benth et al. [7], Šaltytė Benth et al. [25], and contrast it to the approach of Campbell and Diebold [14].

As observed by Campbell and Diebold [14] for US temperature data, and later confirmed for Swedish, Norwegian and Lithuanian temperatures (see above-cited papers), there is a clear seasonality in the temperature variations after removing trend, seasonal variations and AR effects from the data. The model for temperature proposed by Benth and Šaltytė Benth contains (linear) trend, seasonal component, low-order AR process and a seasonal variance component describing the remaining heteroskedasticity in temperature residuals. It differs from the model of Campbell and Diebold [14] first of all in its simplicity. Beside trend and seasonal component, Campbell and Diebold 
propose to use a high-order AR time series model with seasonal generalized AR conditional heteroskedesticity $(\mathrm{GARCH})$ model for the residuals. We are able to show that the model by Benth and Šaltytė Benth is sophisticated enough to explain the basic stylized facts of temperatures just as well as the parameter-intensive alternative proposed by Campbell and Diebold [14].

The contribution of this paper is threefold. First, we critically review the process of modelling the temperatures. In this paper we promote a stepwise procedure used in $[5,7,25]$, where one models and estimates each component step-by-step. This turns out to be advantageous in order to build a confident model explaining the various stylized facts of temperature. In particular, such an approach leads to a very low-order AR structure in the temperature dynamics, in contrast to the approach of Campbell and Diebold [14] suggesting to use an AR model with 25 lags. We argue that one can model temperature dynamics equally good using $\mathrm{AR}(3)$ and show that a simpler model explains the temperature evolution very well. A bigger empirical analysis also shows that the model by Benth and Šaltyte Benth explains extremely good the historical index values of CAT, HDD and CDD.

As a second contribution, we discuss the major role played by the mean of temperature in the context of weather derivatives. The main factor explaining the indices CAT, HDD and CDD turns out to be the seasonal mean temperature, as we demonstrate later on. This is not surprising, taking into consideration the relatively strong mean reversion of temperatures along with indices averaging temperatures over intervals like months. In the model proposed by Benth and Šaltytė Benth, the seasonal mean is modelled explicitly, and is directly estimated from temperature observations. In this way one 
obtains a confident model for the seasonality of temperature. Campbell and Diebold [14] choose to model it indirectly, estimated together with all the other parameters in the model. In the model by Benth and Šaltytė Benth, one regresses the deasonalized temperatures on deseasonalized temperatures, that is, the AR structure is modelled after removing the seasonal mean. Campbell and Diebold [14] choose to regress today's deseasonalized temperature on the temperature in previous days. Their seasonal function will then not be the seasonal mean, but merely a seasonal component. We demonstrate how one can compute the seasonal mean from the model of Campbell and Diebold, involving the AR parameters and thus leading to potentially increased uncertainty in parameter estimates.

The third contribution of the paper is a multiplicative seasonal stochastic volatility model. Instead of using an additive GARCH process in modelling the seasonal heteroskedastic residuals as Campbell and Diebold [14] do, we suggest using a product between a seasonal deterministic function and a classical GARCH process instead. With a multiplicative structure one avoids potential problems related to the positivity of variance. Moreover, no new estimation procedure is required to estimate the GARCH component, leading to a model which is simpler to fit and therefore more practically applicable.

The paper is organized as follows. First, we state the model for the daily temperature variations and discuss in detail the different components of it. Then we describe the data and estimate the model. Next, we validate the proposed model on out-of-sample data and apply it for forecasting different temperature indices. We end our paper by discussing continuous-time models and weather derivatives pricing. 


\section{A general model for temperature dynamics}

We present a general time series model for the temperature dynamics, which is nesting many of the existing models. For modelling of temperature, we suggest to use a time series decomposition approach, where the time series is decomposed into different components like trend, seasonality, an AR process (so-called cyclic component) and residual term, all appearing in observed data simultaneously. By estimating and eliminating different components of time series step-by-step and examining all intermediate residuals, one gets a good insight into the data structure and is likely able to come up with a precise model.

We consider the following model for temperature (see Benth et al. [8]):

$$
Z(t)=\mu(t)+\varepsilon(t)
$$

where $\mu(t)$ and $\varepsilon(t)$ denote, respectively, the mean and the residual process at time $t=1, \ldots, \tau$. Here

$$
\mu(t)=S(t)+\sum_{i=1}^{p} \alpha_{i}(Z(t-i)-S(t-i)),
$$

where $S(t)$ is a deterministic function and $\alpha_{i}, i=1, \ldots, p$, are the parameters of the $\operatorname{AR}(p)$ process. The deterministic function $S(t)$ plays the role of the long-term average of the temperature, towards which the temperature mean reverts due to the AR structure. Another way to represent (2) is to write

$$
Z(t)-S(t)=\sum_{i=1}^{p} \alpha_{i}(Z(t-i)-S(t-i))+\varepsilon(t),
$$

where it is assumed that the deseasonalized temperature follows an $\operatorname{AR}(p)$ process, i.e. todays' deseasonalized temperature is regressed on the $p$ previous 
days' deseasonalized temperatures. As long as the residual process $\varepsilon(t)$ has mean zero, we observe that the expected temperature $\widehat{Z}(t)=\mathbb{E}[Z(t)]$ follows the recursion

$$
\widehat{Z}(t)-S(t)=\sum_{i=1}^{p} \alpha_{i}(\widehat{Z}(t-i)-S(t-i)),
$$

and under stationarity hypothesis of the AR coefficients $\alpha_{i}$ we have that the stationary mean of $Z(t)$ is $S(t)$. Thus, taking the representation (2) and (3) of the temperature dynamics gives a natural interpretation of $S(t)$ as the temperature seasonality. In the sequel, we will frequently refer to this as the seasonal mean function of temperature.

Some authors have modelled temperatures as a mean reversion towards a seasonal mean level. Dornier and Querel [17] suggest an AR(1) structure for temperatures observed at Chicago O'Hare airport, whereas Alaton et al. [1] use a similar model for temperatures in Bromma, Sweden. Brody et al. [11] suggest to model temperatures in central England by such a model, followed by Benth and Saltyte-Benth [4] for temperatures collected in several cities in Norway and Mraoua and Bari [23] for observations in Casablanca, Marocco. Cao and Wei [12] use the approach to model temperature evolution in five US cities. Higher-order AR models have been proposed and analysed by Benth et al. $[25,7]$ for data in Sweden and Lithuania. Recently, such models have been extended to Berlin data and several Asian cities by Härdle and Lopez Cabrera [19] and Benth et al. [9].

The reason for emphasizing the structure (3) is that Campbell and Diebold [14] propose a slightly different representation. They choose the mean process 
$\mu(t)$ to be

$$
\mu(t)=\widetilde{S}(t)+\sum_{i=1}^{p} \alpha_{i} Z(t-i)
$$

for a deterministic function $\widetilde{S}(t)$. In this case, the stationary mean temperature will not be given as $\widetilde{S}(t)$, and the role of $\widetilde{S}$ does not have any physical intepretation other than being a deterministic component in the AR temperature dynamics. In fact, in Campbell and Diebold [14], $\widetilde{S}(t)$ is called the seasonal component or seasonality of the temperature dynamics, and not the seasonal mean function of temperatures, a distinction which is very important for both the interpretation and the application of the model. Chang et al. [15] adopt the model of Campbell and Diebold in their study for five US cities (for the same as in Cao and Wei [12]), while Svec and Stevenson [27] use it to fit Sidney temperature data. Recently, Papazian and Skiadopoulos [24] compared the model of Campbell and Diebold with other models in a bigger empirical study consisting of 10 US and five European cities.

Taking $\mu(t)$ as in (3), we can retwrite it as

$$
\mu(t)=S(t)-\sum_{i=1}^{p} \alpha_{i} S(t-i)+\sum_{i=1}^{p} \alpha_{i} Z(t-i),
$$

leading us to

$$
\widetilde{S}(t)=S(t)-\sum_{i=1}^{p} \alpha_{i} S(t-i) .
$$

Thus, $\mu(t)$ defined as in (3) implies the representation of Campbell and Diebold in (5) in mathematical terms. However, as we shall discuss in subsection 2.1, it is advantageous to choose the former when modelling the temperatures. 
We assume that the seasonal mean function $S(t)$ has the form

$$
S(t)=a_{0}+a_{1} t+\sum_{j=1}^{J} b_{1 j} \cos \left(2 \pi j\left(t-b_{2 j}\right) / 365\right) .
$$

The level of the long-term average temperature is $a_{0}$, while the trend $a_{1} t$ ensures stationarity in temperature time series, since the temperatures might have risen due to global warming and urbanisation, say. The sum of trigonometric functions explains the seasonal variation in temperature, varying with the colder and warmer periods of the year. We know from Thm 8.20 in Folland [18] that the set $\{\exp (2 \pi \kappa \mathrm{i})\}_{\kappa \in \mathbb{Z}}$ is an orthonormal basis in $L^{2}(\mathbf{T})$ for $\mathbf{T}$ being the torus on the real line. Thus, we can approximate any square integrable periodic function by a sum of trigonometric functions as in (7) arbitrarily good. However, we have a very low order of $J$ in mind, in fact $J=1$ seems to be enough for capturing the seasonal behaviour in most temperature data series. We remark in passing that other temperature seasonality functions have been recently proposed by Härdle et al. [20] in relation to a study of Asian cities.

Note that by using the fundamental identity

$$
\cos (x-y)=\cos (x) \cos (y)+\sin (x) \sin (y)
$$

we find that $S(t)$ can equivalently be rewritten as

$$
\begin{aligned}
S(t)=a_{0} & +a_{1} t+\sum_{j=1}^{J} b_{1 j} \cos \left(2 \pi j b_{2 j} / 365\right) \cos (2 \pi j t / 365) \\
& +\sum_{j=1}^{J} b_{1 j} \sin \left(2 \pi j b_{2 j} / 365\right) \sin (2 \pi j t / 365) .
\end{aligned}
$$


Letting $\widetilde{b}_{1 j}=b_{1 j} \cos \left(2 \pi j b_{2 j} / 365\right)$ and $\widetilde{b}_{2 j}=b_{1 j} \sin \left(2 \pi j b_{2 j} / 365\right)$, we have that $S(t)$ is of the same form as the seasonal component $\widetilde{S}(t)$ used in the Campbell and Diebold model [14].

\subsection{Recovering the seasonal mean function from the seasonal component}

In the model of Campbell and Diebold, the seasonality of the temperature is modelled implicitly through the seasonal component $\widetilde{S}(t)$ and the AR structure, as we recall from (6). We now show how the true seasonal function $S(t)$ can be recovered in the situation where the seasonal component in the model of Campbell and Diebold is known. For this purpose, we suppose that

$$
\widetilde{S}(t)=\widetilde{a}_{0}+\widetilde{a}_{1} t+\widetilde{b}_{1} \cos (2 \pi t / 365)+\widetilde{b}_{2} \sin (2 \pi t / 365) .
$$

The estimates of the parameters $\widetilde{a}_{0}, \widetilde{a}_{1}, \widetilde{b}_{1}$ and $\widetilde{b}_{2}$ are found from data. Admittedly, in Campbell and Diebold [14] a series of trigonometric function with yearly, half-yearly and quarterly frequencies (choosing $J=3$, in fact) are used. But for the following argument, and motivated from the emprical study to come, we focus on the simple case here.

Next, we define the "true" seasonal mean function $S(t)$ to be

$$
S(t)=a_{0}+a_{1} t+b_{1} \cos (2 \pi t / 365)+b_{2} \sin (2 \pi t / 365) .
$$

Our goal is to recover $a_{0}, a_{1}, b_{1}$ and $b_{2}$ from the known quantities $\widetilde{a}_{0}, \widetilde{a}_{1}, \widetilde{b}_{1}$ and $\widetilde{b}_{2}$, respectively. By applying (6) with the fundamental identities for trigonometric functions

$\cos (2 \pi(t-k) / 365)=\cos (2 \pi k / 365) \cos (2 \pi t / 365)+\sin (2 \pi k / 365) \sin (2 \pi t / 365)$, 
and

$\sin (2 \pi(t-k) / 365)=\cos (2 \pi k / 365) \sin (2 \pi t / 365)-\sin (2 \pi k / 365) \cos (2 \pi t / 365)$,

we find the following set of equalities after collecting the terms for level, trend and the trigonometric functions:

$$
\begin{aligned}
& a_{0}=\widetilde{a}_{0}+a_{0} \sum_{i=1}^{p} \alpha_{i}-a_{1} \sum_{i=1}^{p} i \alpha_{i}, \\
& a_{1}=\widetilde{a}_{1}+a_{1} \sum_{i=1}^{p} \alpha_{i}, \\
& b_{1}=\widetilde{b}_{1}+b_{1} \sum_{i=1}^{p} \alpha_{i} \cos (2 \pi i / 365)-b_{2} \sum_{i=1}^{p} \alpha_{i} \sin (2 \pi i / 365), \\
& b_{2}=\widetilde{b}_{2}+b_{1} \sum_{i=1}^{p} \alpha_{i} \sin (2 \pi i / 365)+b_{2} \sum_{i=1}^{i} \alpha_{i} \cos (2 \pi i / 365) .
\end{aligned}
$$

Solving for the unknown parameters, we find

$$
\begin{aligned}
a_{0} & =\frac{\widetilde{a}_{0}\left(1-\sum_{i=1}^{p} \alpha_{i}\right)-\widetilde{a}_{1} \sum_{i=1}^{p} i \alpha_{i}}{\left(1-\sum_{i=1}^{p} \alpha_{i}\right)^{2}}, \\
a_{1} & =\frac{\widetilde{a}_{1}}{1-\sum_{i=1}^{p} \alpha_{i}}, \\
b_{1} & =\frac{\widetilde{b}_{1}\left(1-\sum_{i=1}^{p} \alpha_{i} \cos (2 \pi i / 365)\right)-\widetilde{b}_{2} \sum_{i=1}^{p} \alpha_{i} \sin (2 \pi i / 365)}{\left(\sum_{i=1}^{p} \alpha_{i} \sin (2 \pi i / 365)\right)^{2}+\left(1-\sum_{i=1}^{p} \alpha_{i} \cos (2 \pi i / 365)\right)^{2}}, \\
b_{2}= & \frac{\widetilde{b}_{1} \sum_{i=1}^{p} \alpha_{i} \sin (2 \pi i / 365)+\widetilde{b}_{2}\left(1-\sum_{i=1}^{p} \alpha_{i} \cos (2 \pi i / 365)\right)}{\left(\sum_{i=1}^{p} \alpha_{i} \sin (2 \pi i / 365)\right)^{2}+\left(1-\sum_{i=1}^{p} \alpha_{i} \cos (2 \pi i / 365)\right)^{2}} .
\end{aligned}
$$

Note the occurrence of the AR parameters $\alpha_{i}$ in all expressions. Thus, in order to recover the true seasonality function we must know the AR parameters as well. These are estimated together with $\widetilde{a}_{0}, \widetilde{a}_{1}, \widetilde{b}_{1}$ and $\widetilde{b}_{2}$ in the model of Campbell and Diebold, and are therefore affected by an estimation error. In order to find $a_{0}$, say, we need $p+2$ estimated parameters, all with uncertainty. This makes the estimate of $a_{0}$ very uncertain. To get a feeling 
for what happens, we apply the estimates for the AR parameters found for Stockholm data (see next Section for the estimation procedure). The figures are $\alpha_{1}=0.96, \alpha_{2}=-0.25$ and $\alpha_{3}=0.12$ (see Table 1 ). This gives that $\widetilde{a}_{1}$ is only $17 \%$ of true trend increase $a_{1}$. Since $a_{1}$ is typically very small (in fact, it was estimated to be 0.0001 for the Stockholm data, see next Section), we run the risk of getting an insignificant estimate of $\widetilde{a}_{1}$, even if the trend is significant.

It is highly important to have an accurate estimate of the temperature seasonality function $S(t)$ in applications to weather markets. Computing it from the seasonal component may lead to wrong specifications, including uncertainty in the estimates. Moreover, when applying a stepwise estimation procedure where one first finds the seasonality function, one reveals the true structure of it. Another aspect is in the prediction of indices. Taking, for example, the CAT index defined in (1), the seasonality function $S(t)$ will be the dominating factor. This can be seen from the discussion leading to (4), where $S(t)$ was shown to be the long-term temperature mean level, and simple summation reveals that the mean long-term level of the CAT will simply be $\sum_{t=T_{1}}^{T_{2}} S(t)$ when the measurement period is $\left[T_{1}, T_{2}\right]$. By mean reversion, long measurement periods will imply that the CAT index is essentially equal to the aggregation of the seasonality function. We will return to this in Section 4 on model validation.

\subsection{The residual process}

It is a well known fact that the temperature residuals are not independent identically distributed normal random variables. Alaton et al. [1] observed 
in their analysis of Stockholm temperatures using an AR(1) model that the "volatility" of temperature varied with the seasons over the year. They proposed to model the volatility of each month as a monthly average emprical variance. Further, it was observed by Campbell and Diebold [14] that the autocorrelation function (ACF) of the squared residuals in many US cities has a seasonal structure. The same observation was made for several locations in Norway and Lithuania in the papers Benth and Šaltytè Benth [4] and Šaltytė Benth et al. [25], respectively, for German temperature data in Härdle and Lopez Cabrera [19], and for Asian data in Benth et al. [9]. Moreover, a characteristic seasonal pattern for the daily variance of temperature was observed (see Benth et al [5] for a detailed discussion in connection with Stockholm temperature data). In addition, in Benth et al. [4] and [5] a small GARCH effect in the ACF of the squared residuals after explaining the seasonality in the daily variance was observed, but not modelled.

Motivated by the above studies, we assume that the residual process $\varepsilon(t)$ is of the following form

$$
\varepsilon(t)=\sigma(t) \delta(t)
$$

where $\sigma(t)$ is a seasonally varying stochastic process (a seasonal stochastic volatility), and $\delta(t)$ is a zero-mean temporally independent Gaussian random process with standard deviation equal to one.

As observed in, for example, Benth et al. [5] (see above for more references), the variance of the temperature residuals demonstrates a rather distinctive seasonal pattern, which we model by $\sigma^{2}(t)=\sigma_{\mathrm{BSB}}^{2}(t)$, with

$$
\sigma_{\mathrm{BSB}}^{2}(t)=c_{1}+\sum_{k=1}^{K}\left(c_{2 k} \cos (2 k \pi t / 365)+c_{2 k+1} \sin (2 k \pi t / 365)\right) .
$$


In Benth et al. [4, 7], the choice of $K=3$ turned out to give a very good fit to the yearly seasonal pattern of the residual variance. However, there are examples of locations where the residual variance is varying very little throughout the year (see, for example, Benth et al. [4] for some Norwegian cities and Campbell and Diebold [14] for the US cities Las Vegas and Portland). In such a case it is natural to choose $K=0$, that is, to assume a constant volatility. Of course, there may also exist cities where a higher order $K$ might be needed.

As already mentioned, in many locations one finds signs of GARCH effects in the residuals after removing the influence of $\sigma_{\mathrm{BSB}}(t)$ (that is, in the data $\left.\varepsilon(t) / \sigma_{\mathrm{BSB}}(t)\right)$. Such effects are minor, but to explain them in the proposed model it is natural to assume that $\sigma(t)=\sigma_{\mathrm{BSB}}(t) \sigma_{\mathrm{GARCH}}(t)$ with

$$
\sigma_{\mathrm{GARCH}}^{2}(t)=\beta_{1} \delta^{2}(t-1)+\beta_{2} \sigma_{\mathrm{GARCH}}^{2}(t-1) .
$$

In conclusion, we assume that the residual process $\varepsilon(t)$ as in (9) is defined as

$$
\varepsilon(t)=\sigma_{\mathrm{BSB}}(t) \sigma_{\mathrm{GARCH}}(t) \delta(t),
$$

with $\sigma_{\mathrm{BSB}}(t)$ defined in $(10)$ and $\sigma_{\mathrm{GARCH}}(t)$ in $(11)$.

In Campbell and Diebold [14], a different model is considered. They explain the seasonality and GARCH effect in temperature volatility by an additive seasonal GARCH model for $\sigma(t)$ of the form

$$
\sigma_{\mathrm{CD}}^{2}(t)=\sigma_{\mathrm{BSB}}^{2}(t)+\beta_{1} \delta^{2}(t-1)+\beta_{2} \sigma_{\mathrm{CD}}^{2}(t-1) .
$$

In view of the positivity of the variance, it seems more natural to consider a multiplicative structure of the seasonality and GARCH effect in the temperature volatility, rather than an additive one. It is simple to reveal the true 
seasonality and GARCH effects observed in residual variance using a multiplicative approach, and positivity of the variance is naturally preserved. In addition, in the estimation of the multiplicative model as we suggest, no new estimation procedures are required for the GARCH part.

We remark that Benth et al. [9] are discussing the volatility model of Campbell and Diebold [14] in connection with a study of Asian temperatures using a CAR model. Recently, Härdle et al. [20] considered a local adaptive modelling approach to find at each time point an optimal smoothing parameter to locally estimate the seasonality of the volatility. This approach refines the modelling of $\sigma_{\mathrm{BSB}}(t)$.

\section{Empirical analysis of temperature data}

We have available temperature observations from Stockholm, Sweden, in the period January 1, 1961 to May 25, 2006, resulting in 16,570 records after the observations made on Februrary 29 in all leap years are removed. We leave out the data starting January 1, 2005 and lasting to the end of observation period (out-of-sample data set consisting of 510 observations) for model validation. We thus deal with 16,060 data points (in-sample) when estimating the model.

The average in-sample temperature in Stockholm is $7.2^{\circ} \mathrm{C}$ with standard deviation equal to $8.2^{\circ} \mathrm{C}$ and range $(-23.6,28.7)$. In Fig. 1 we plot a snapshot of the first five years of observations together with the histogram and ACF for the total dataset. We observe a clear seasonal pattern in both the time series and ACF plots for the temperatures. The bimodality in the histogram is also 
appearing due to the cold winters and rather mild summers in Stockholm. In addition, we observe a small left skewness in the data (equal to -0.10) and a negative kurtosis (-0.67).
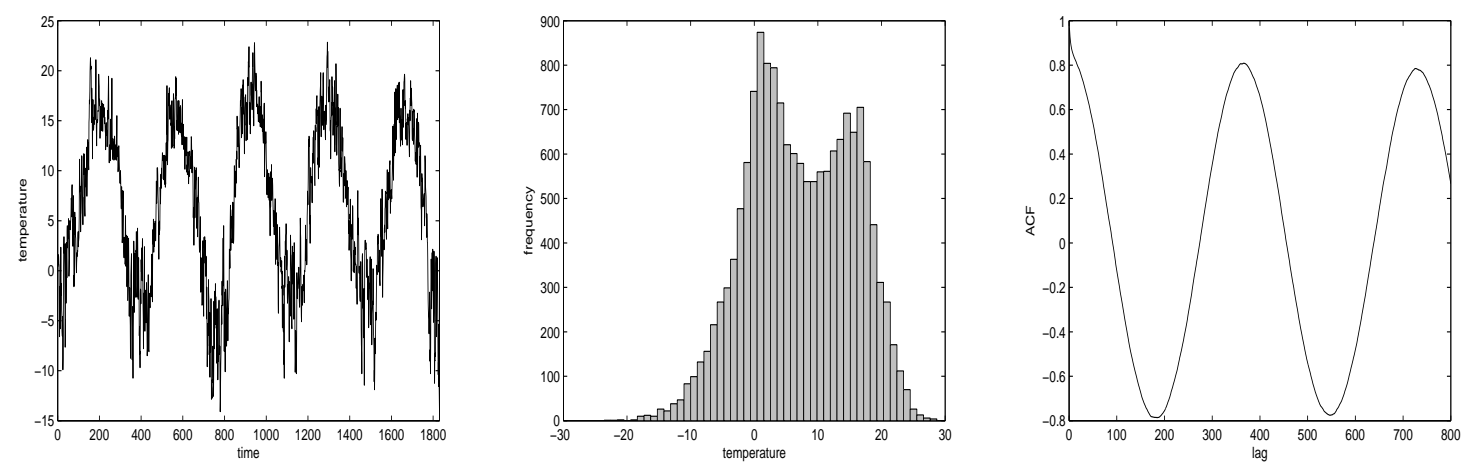

Figure 1: Snapshot of five years of temperatures, histogram and ACF of temperatures in Stockholm.

We now proceed to estimation of the proposed temperature model. We are going to estimate the different components of the model step-by-step and carefully examine the resulting residuals after each component has been eliminated. In this way we want to reveal the true characteristics of the temperature dynamics.

We first estimate the seasonality function $S(t)$ defined in (7). This is done by standard least squares approach, the usual way to identify a mean function. We implemented the least squares estimation procedure by resorting to the built-in function nlinfit in Matlab. The values of the estimates are: $a_{0}=6.37, a_{1}=0.0001, b_{1}=10.44$, and $b_{2}=-161.17$, all significant at the level of $5 \%$ (although the significance level must be treated with care because of non-normally distributed and autocorrelated temperature data). 
The value of the estimated linear slope shows that the temperature in Stockholm has risen approximately $1.5^{\circ} \mathrm{C}$ in the considered period. This finding is in line with observations made by other authors. Our trend-seasonal function $S(t)$ with only four parameters explains $81.1 \%$ of the variation in temperature time series. Removing the estimated seasonal mean function $S(t)$ from the data resulted in a unimodal histogram. The trend-seasonal function with eight parameters, suggested by Campbell and Diebold [14], explains $81.6 \%$ of the variation in temperature in Stockholm, a very small increase in performance although a doubling in the number of parameters. We note that according to Campbell and Diebold, their trend-seasonal function explains about $90 \%$ of the variation in data for the USA cities considered in their study.

The ACF values of detrended and deseasonalized data start at around 0.8 and stay rather high (and of course significant) for many lags (see Fig. 2, left). Clearly, there are memory effects in the data not captured by the seasonal mean function $S(t)$. The analysis of the PACF (see Fig. 2, right) suggests an $\mathrm{AR}(3)$ process to explain the AR pattern in the residuals. The parameter estimates of the $\operatorname{AR}(3)$ process are given in Table 1 together with the $R^{2}$ value. The estimated parameters are all significant at the $5 \%$ level. The model fit is already very good, with $R^{2}$ of $94.1 \%$.

The choice of an AR(3) model is in agreement with many other studies of temperature data. Šaltytė Benth et al. [25] analyse Lithuanian temperature data and argue empirically for an AR(3) model, while Härdle and Lopez Cabrera [19] find the same for Berlin temperatures. In Benth et al. [9] several Asian cities are considered, and the choice of an $\mathrm{AR}(3)$ is made based on 

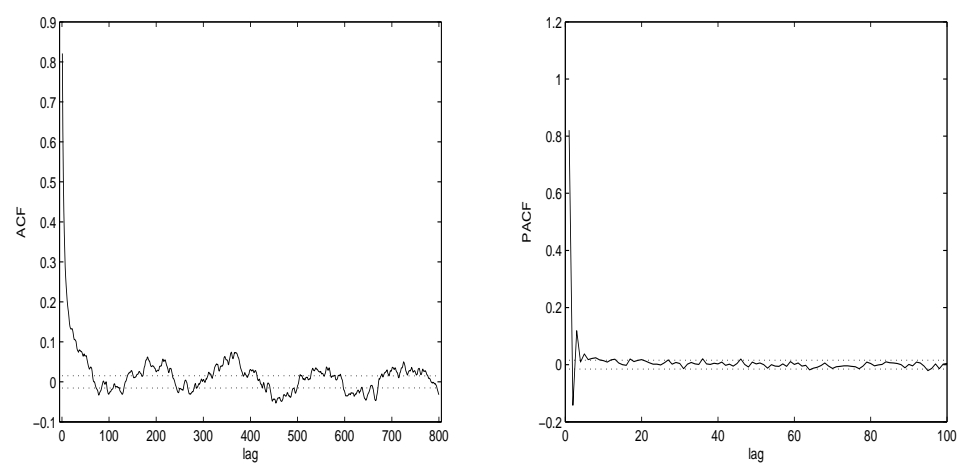

Figure 2: $\mathrm{ACF}$ and PACF of deseasonalized and detrended temperatures.

empirical arguments. Campbell and Diebold [14] claim that $\operatorname{AR}(25)$ is the optimal choice in their study of US temperature data based on the Akaike's Information Criterion (AIC). Interestingly, Cao and Wei [12] apply an AR(3) model for the cities Atlanta, Chicago and Philadelphia, which are also part of the study of Campbell and Diebold. ${ }^{1}$ Note that the two studies are not directly comparable, since Cao and Wei consider an AR model for deseasonalized data. It is our experience that to choose 25 lags to model the temperature is far above what is necessary from a statistical point of view. Furthermore, it is not clear if there is any meteorological explanation for why today's temperature should explicitly depend on what happened 25 days ago. We remark that for Stockholm temperature data, the value of AIC is smaller for the $\operatorname{AR}(3)$ model than for the $\operatorname{AR}(25)$ model, which is not in line with the conclusions of Campbell and Diebold [14]. Moreover, most of the lags become insignificant when estimating an $\mathrm{AR}(25)$ model, and the $R^{2}$ was not

\footnotetext{
${ }^{1}$ In the older version of the paper of Campbell and Diebold [13], the cities are fully overlapping with those five studied by Cao and Wei [12]. Cao and Wei use an AR(3) for all cities.
} 
Table 1: Estimates of parameters of the AR(3) process.

\begin{tabular}{rrrr}
$\alpha_{1}$ & $\alpha_{2}$ & $\alpha_{3}$ & $R^{2}(\%)$ \\
\hline 0.96 & -0.25 & 0.12 & 94.1 \\
\hline
\end{tabular}

improved as compared with the AR(3) model.

For pure comparison, we took the function $\widetilde{S}(t)$ in the representation of $\mu(t)$ in (5) and interpreted it as the seasonal mean. As suggested by Cambell and Diebold [14], we should regress the deseasonalized temperatures on the temperatures. For the seasonal function $S(t)$ and an $\operatorname{AR}(3)$ model, the $R^{2}$ value became by almost $10 \%$ lower than in the case of the model specification proposed in this paper. Moreover, we did not get much better fit by choosing $\mathrm{AR}(25)$ as suggested by Campbell and Diebold; most of the parameters are insignificant (those significant ones are very small in value, except for the first three). By increasing the number of AR parameters from three to 25 we managed to increase the value of $R^{2}$ by $5.4 \%$ (from $84.6 \%$ to $89.9 \%$ ). Note that it is still lower than the one obtained with $\operatorname{AR}(3)$ model on deseasonalized data.

Let us look at the effects on the residuals when using the "wrong" model specification above. In Fig. 3, we show the ACF for both types of model specifications. The left and the right panels present, respectively, the ACF for residuals and the ACF for squared residuals for the proposed model. In the middle plot, the ACF for the residuals for the model of Campbell and Diebold [14] where the function $\widetilde{S}(t)$ is intepreted as the seasonal mean, is presented. All three plots are obtained after $\operatorname{AR}(3)$ process has been estimated and eliminated from the data. The ACF of the "wrong" specification is again 
demonstrating seasonality. In addition, data are highly autocorrelated. In other words, by regressing the deseasonalized data on original temperatures, we impose the seasonality back into the data. When looking at the ACF for residuals obtained with our approach, we see that there is no seasonality left and residuals are basically uncorrelated. As is apparent, the inclusion of $\widetilde{S}(t)$ in Campbell and Diebold [14] is not to be intepreted as the seasonal mean of the temperature, and doing so leads to highly unreasonable effects. This demonstrates the advantage of the decomposition approach used; one has full controll over the effects of each model component. This makes it possible to reveal potential misspecification of the model.
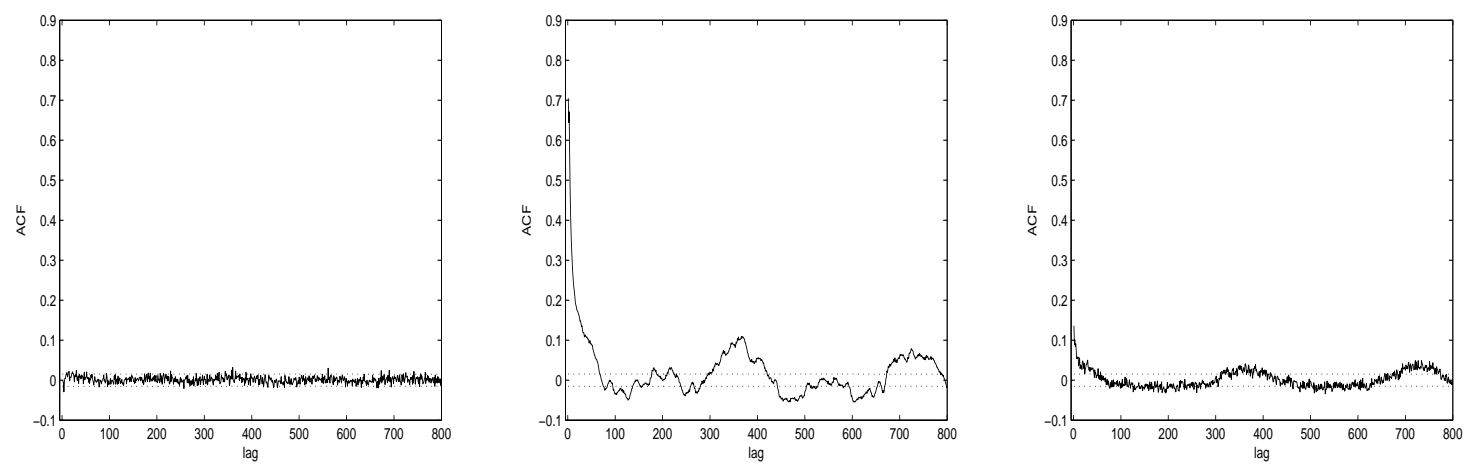

Figure 3: ACF of residuals (detrended and deseasonalized DAT after AR(3) has been eliminated) (left), squared residuals (right) and residuals when CD specification is used (middle).

The ACF for squared residuals (right-hand plot in Fig. 3) demonstrates a clear seasonal pattern, calling for more sophisticated models than just a white noise process. We consider a seasonal volatility model with and without a multiplicative $\operatorname{GARCH}(1,1)$, as defined in (11) and (10), respectively. 
Table 2: Estimates of parameters of the $\sigma_{\mathrm{BSB}}^{2}(t)$ in Stockholm.

\begin{tabular}{rrrrrrr}
$c_{1}$ & $c_{2}$ & $c_{3}$ & $c_{4}$ & $c_{5}$ & $c_{6}$ & $c_{7}$ \\
\hline 4.024 & 1.177 & 0.695 & 0.781 & -0.162 & 0.201 & 0.436 \\
\hline
\end{tabular}

To estimate the volatility model, we first compute the daily empirical variance by averaging the values of the squared residuals of the particular day over all years. This results in 365 data points, each calculated from 45 residuals. In Fig. 4 we have plotted the fitted truncated Fourier function (10) with the empirical daily variances, where we observe the clear seasonal pattern present in the data. The parameters of the fitted function (10) are given in Table 2. From Fig. 4 we see that the temperature fluctuations in the cold (or HDD) season are considerably higher than those during the warm (CDD) season. Furthermore, the variations seem to be lower in spring and autumn, than in the summer. These observations are consistent with Cao and Wei [12] and Campbell and Diebold [14].

Neither residuals nor squared residuals obtained after $\sigma_{\mathrm{BSB}}(t)$ was eliminated from the data reveal any seasonal pattern (not shown). The ACF plots for residuals and squared residuals in Fig. 5 (top row) confirm that basically all the seasonality is explained by the model. On the other hand, from the ACF of the squared residuals we observe a small GARCH effect not accounted for. Motivated by this, we fit the model in (11) as well, where the seasonal variance function $\sigma_{\mathrm{BSB}}^{2}(t)$ is multiplied by a $\operatorname{GARCH}(1,1)$ process. The parameters of the $\operatorname{GARCH}(1,1)$ model were estimated to be $\beta_{1}=0.06$ and $\beta_{2}=0.93$. As it is seen from the Fig. 5 (bottom row), the GARCH effect is clearly explained. In order to demonstrate how well our model is capturing 


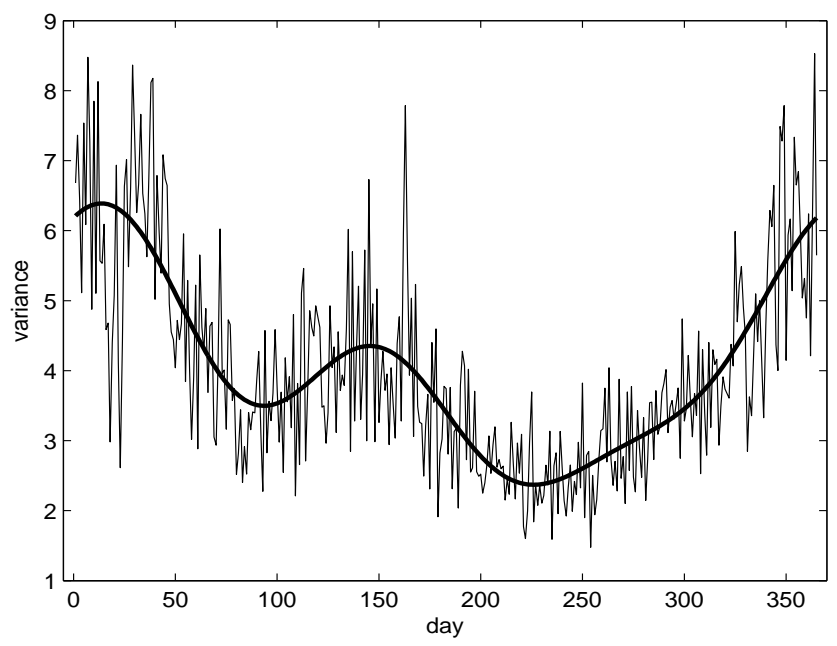

Figure 4: Daily variances of final residuals together with the fitted function $\sigma_{\mathrm{BSB}}^{2}(t)$.

the seasonal variations in the residuals, we plot in Fig. 6 the squared residuals after trend-season component and $\operatorname{AR}(3)$ process have been eliminated from temperature data together with the fitted $\sigma_{\mathrm{BSB}}^{2}(t) \sigma_{\mathrm{GARCH}}^{2}(t)$ function.

Final residuals are demonstrating clear symmetry (Fig. 7) for both cases of variance functions used. The $p$-values of the Kolmogorov-Smirnov normality test are in both cases around 0.01. With the amount of data we have at hand, it is almost impossible to reach insignificant $p$-values with a test such as Kolmogorov-Smirnov, since even small deviations from normality may have a big influence on the test statistic. The variances of residuals are essentially equal to one, while the means, skewnesses and kurtoses are close to zero, indicating that the distributions are close to normal. 

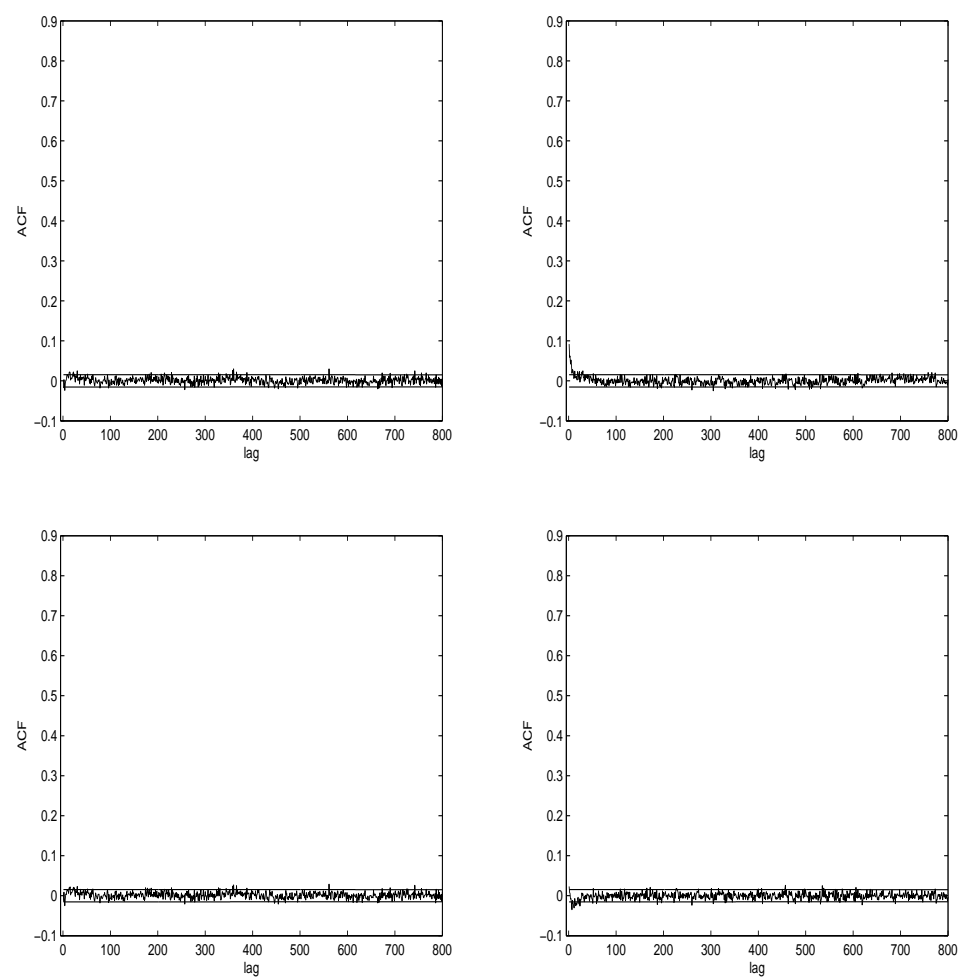

Figure 5: ACF for final residuals (left) and squared residuals (right) after $\sigma_{\mathrm{BSB}}^{2}(t)$ (top row) and $\sigma_{\mathrm{BSB}}^{2}(t) \sigma_{\mathrm{GARCH}}^{2}(t)$ (bottom row) have been eliminated.

\section{Model validation}

For model validation we used 510 out-of-sample observations. To validate the model, one-step-ahead predictions for out-of-sample observations were generated and prediction errors (PE) calculated as differences between the observed and predicted values. PEs were normally distributed ( $p$-value for the Kolmogorov-Smirnov test was 0.61) and not autocorrelated with mean zero and standard deviation of 1.9 .

Further, the prediction intervals (PI) were built by simulating 1000 re- 


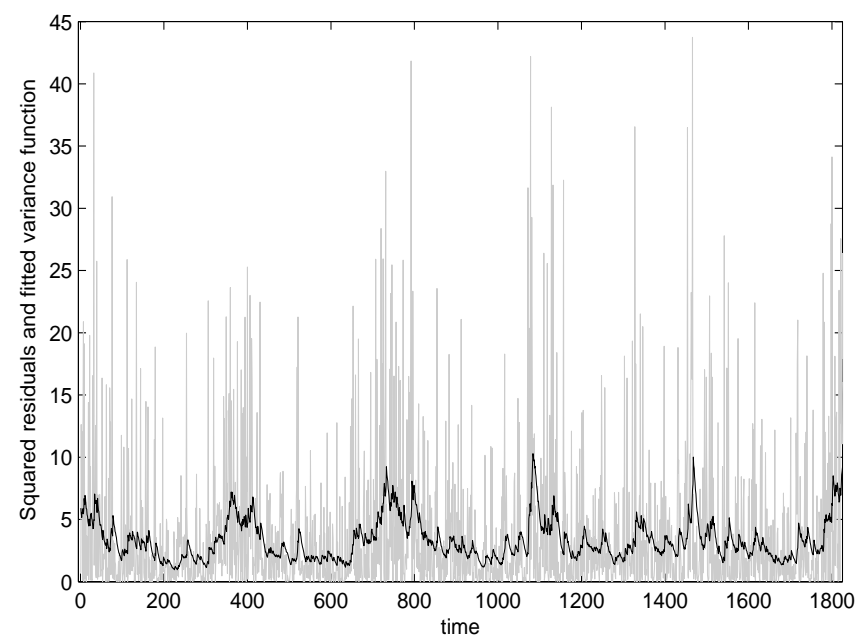

Figure 6: Squared residuals after trend-seasonal function and $\operatorname{AR}(3)$ have been removed together with fitted $\sigma_{\mathrm{BSB}}^{2}(t) \sigma_{\mathrm{GARCH}}^{2}(t)$ function.

alisations of the model by first generating a series of random innovations and then constructing a sequence of values from the model. From this set of simulated observations, we calculated PIs by finding the interval within which the required percentage of future values lie. Various PIs together with the percentage of predicted values outside of them are given in Table 3. For illustrative purposes, we plot in Fig. 8 the observed and predicted values together with the $95 \%$ PI containing $0.39 \%$ of predicted values outside the PI.

Cumulative HDDs and CATs are very relevant for weather derivatives. We use the estimated model for temperature to simulate the cumulative HDDs and CATs for the cold (October 1 through April 30) and warm (April 1 through October 31) periods, respectively, in the years 1961 through 2004. The simulation is performed in the following way. Just as for the model val- 

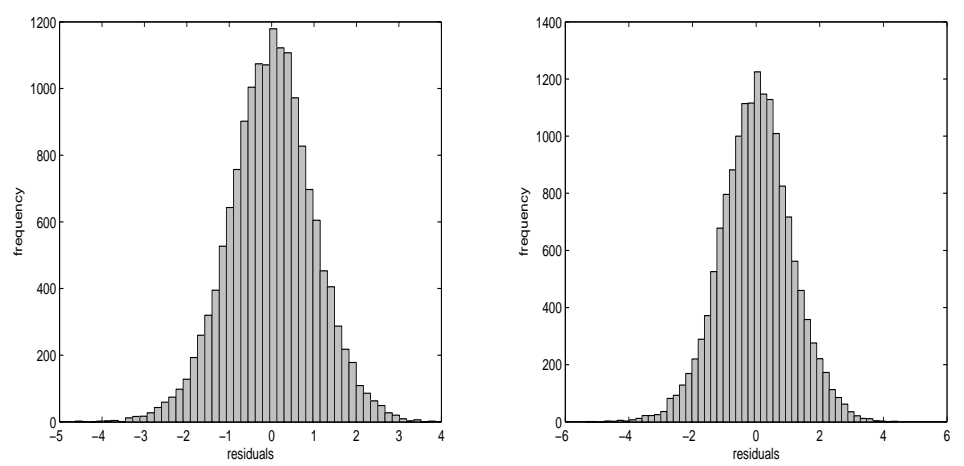

Figure 7: Histograms of final residuals for $\sigma_{1}^{2}(t)$ (left) and $\sigma_{\mathrm{BSB}}^{2}(t) \sigma_{\mathrm{GARCH}}^{2}(t)$ (right).

idation, we first generate a series of innovations from the standard normal distribution in order to construct a sequence of realizations of temperatures from the model. We repeat this process 1000 times. From the simulated series, we calculate cumulative HDDs and CATs for relevant months. To summarize the results of simulation, we aggregate all simulated values into few descriptive statistics and present them in Table 4 and Table 5 together with the corresponding statistics for observed HDDs and CATs. The observed and simulated mean, min and max values of HDD and CAT are close to each other, just validating the proposed model once more. The $95 \%$ confidence intervals (CI) for observed HDDs and CATs are much wider than those for simulated indices. This is because the observed CIs are only based on 45 years of data, while the simulated ones are the result of 1000 iterations.

We have earlier in this paper argued for the importance of the seasonality function $S(t)$ in temperature modelling and weather derivatives pricing. We discuss next how the seasonal function explains the CAT and HDD in- 
Table 3: Various PIs based on percentiles.

\begin{tabular}{rr}
\hline PI & $\%$ \\
\hline$(0.05 ; 99.95)$ & 0 \\
$(2.5 ; 97.5)$ & 0.39 \\
$(16.0 ; 64.0)$ & 13.95 \\
$(20.0 ; 80.0)$ & 21.81 \\
$(30.0 ; 70.0)$ & 39.49 \\
$(40.0 ; 60.0)$ & 69.55 \\
\hline
\end{tabular}

dices. In Fig. 9 (left) we have plotted the CAT index computed from a 30 days window of Stockholm temperatures rolling through the year 2004. In addition, we have calculated the index based solely on the estimated seasonal mean function $S(t)$ in (7). As we see, the CAT index derived from the seasonal mean function follows remarkably close the empirical one. The same observation is made when doing the exercise for the HDD index instead (Fig. 9 (right)). This is seemingly more surprising, however, since the cut-off value is $18^{\circ} \mathrm{C}$ and the daily average rarely goes above this, the HDD index is corresponding to the CAT for large parts of the year.

From a scatter plot of the empirically observed index versus the one computed from the estimated seasonal mean function (Fig. 10), we observe that observed and estimated values for both CAT and HDD show a strong linear dependence. The slope in a linear regression model is close to one for both CAT and HDD. Although the regression analysis may be based on false assumptions, it gives a clear indication of the strong explanatory power of the seasonal function when it comes to index prediction. Note that it is 
Table 4: Descriptive statistics for observed and simulated values of HDD.

\begin{tabular}{rrrrrrr}
\hline Month & & Mean & $95 \%$ CI & Min & Max \\
\hline \multirow{2}{*}{ January } & Observed & 626.2 & $(436.3 ; 816.0)$ & 459.2 & 903.3 \\
& Simulated & 631.4 & $(593.3 ; 669.4)$ & 428.6 & 933.0 \\
\hline Februray & Observed & 572.3 & $(381.3 ; 763.3)$ & 383.4 & 802.6 \\
& Simulated & 570.0 & $(536.6 ; 603.3)$ & 348.5 & 811.1 \\
\hline March & Observed & 536.1 & $(402.3 ; 669.8)$ & 404.4 & 661.7 \\
& Simulated & 533.7 & $(503.8 ; 563.6)$ & 372.1 & 689.7 \\
\hline April & Observed & 383.8 & $(298.7 ; 469.0)$ & 306.1 & 487.0 \\
& Simulated & 378.4 & $(349.8 ; 407.1)$ & 261.0 & 510.9 \\
\hline October & Observed & 324.9 & $(231.7 ; 518.1)$ & 212.2 & 430.1 \\
& Simulated & 322.9 & $(295.8 ; 350.1)$ & 186.7 & 444.7 \\
\hline November & Observed & 459.6 & $(356.9 ; 562.4)$ & 331.5 & 573.9 \\
& Simulated & 459.7 & $(429.7 ; 489.8)$ & 310.9 & 598.0 \\
\hline December & Observed & 580.3 & $(445.5 ; 715.1)$ & 440.3 & 723.7 \\
& Simulated & 584.1 & $(548.5 ; 619.7)$ & 419.6 & 758.3 \\
\hline
\end{tabular}


Table 5: Descriptive statistics for observed and simulated values of CAT.

\begin{tabular}{rrrrrr}
\hline Month & & Mean & $95 \%$ CI & Min & Max \\
\hline \multirow{2}{*}{ April } & Observed & 156.2 & $(71.0 ; 241.4)$ & 53.0 & 233.9 \\
& Simulated & 161.6 & $(132.9 ; 190.3)$ & 29.1 & 279.2 \\
\hline May & Observed & 344.0 & $(253.2 ; 434.7)$ & 272.3 & 445.7 \\
& Simulated & 346.0 & $(314.8 ; 377.2)$ & 234.7 & 486.0 \\
\hline \multirow{2}{*}{ June } & Observed & 478.4 & $(394.5 ; 562.2)$ & 381.8 & 537.1 \\
& Simulated & 476.1 & $(446.4 ; 505.7)$ & 349.3 & 594.8 \\
\hline \multirow{2}{*}{ July } & Observed & 560.3 & $(456.8 ; 663.8)$ & 457.8 & 685.3 \\
& Simulated & 555.8 & $(529.6 ; 581.9)$ & 427.7 & 695.1 \\
\hline \multirow{2}{*}{ August } & Observed & 529.6 & $(420.1 ; 639.1)$ & 422.8 & 673.8 \\
& Simulated & 527.6 & $(504.0 ; 551.3)$ & 403.2 & 684.3 \\
\hline \multirow{2}{*}{ September } & Observed & 370.2 & $(289.0 ; 451.3)$ & 287.2 & 488.2 \\
& Simulated & 374.9 & $(350.4 ; 399.4)$ & 265.2 & 509.6 \\
\hline \multirow{2}{*}{ October } & Observed & 233.1 & $(140.0 ; 326.3)$ & 127.9 & 345.9 \\
& Simulated & 235.1 & $(208.0 ; 262.3)$ & 113.3 & 371.3 \\
\hline
\end{tabular}




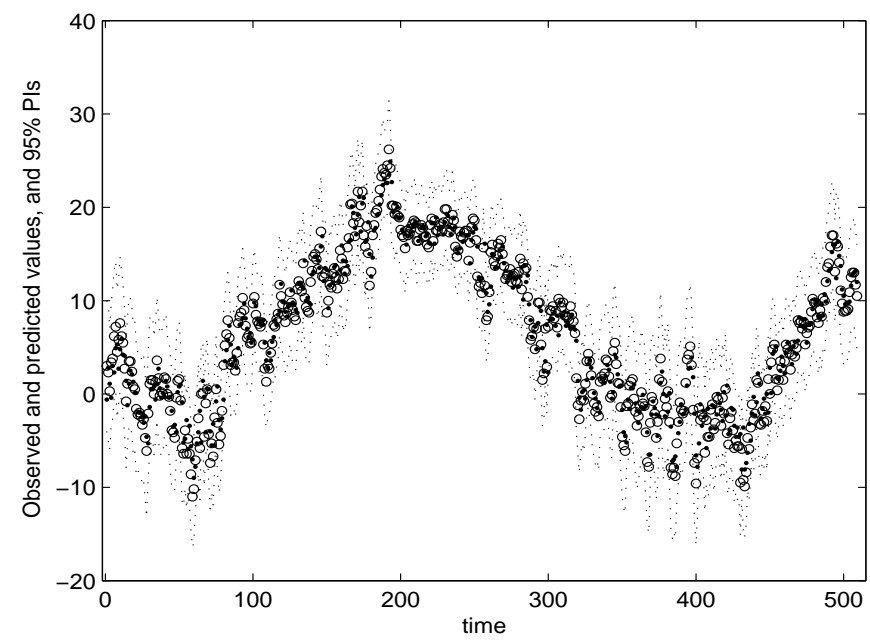

Figure 8: Observed (circles) and predicted (discs) values with 95\% prediction intervals (dotted line).

the relatively strong mean reversion of temperature combined with a linear noise structure which explains the close connection between the seasonality function and the index value. In view of these results, it is clear that emphasis must be put on the seasonality function when modelling temperature dynamics.

\section{A discussion of continuous-time models and weather derivatives pricing}

Admittedly, most of the models for the temperature dynamics mentioned in this paper are stated as continuous-time stochastic processes, with the exception of the model of Campbell and Diebold [14]. For example, in Benth 

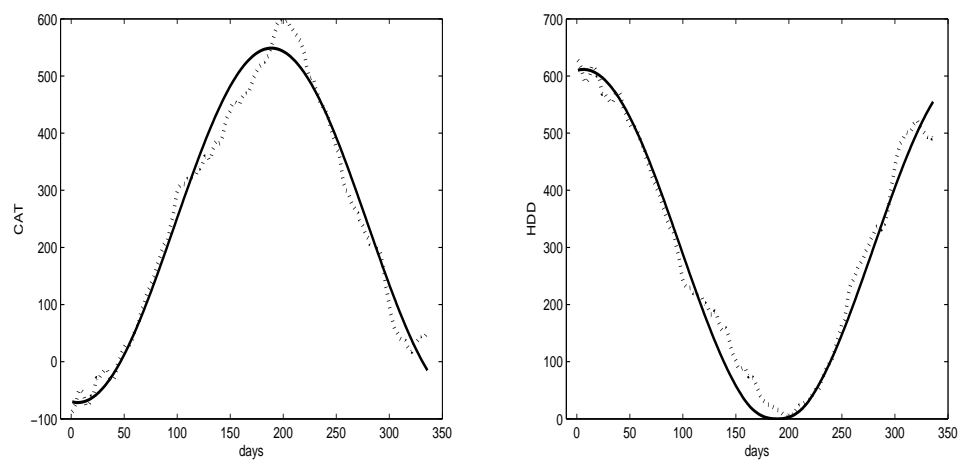

Figure 9: Observed and estimated (solid line) CAT (left) and HDD (right) indices for year 2004 calculated from a 30 days window.

and Šaltytė Benth [4], the dynamics of the deseasonalized temperature is assumed to follow an Ornstein-Uhlenbeck process

$$
d \widetilde{Z}(t)=-\alpha \widetilde{Z}(t) d t+\sigma_{\mathrm{BSB}}(t) d B(t)
$$

where the temperature is defined as $Z(t)=S(t)+\widetilde{Z}(t), \alpha>0$ is a positive constant measuring the speed of mean reversion and $B$ is a Brownian motion defined on a filtered probability space $\left(\Omega, \mathcal{F}, \mathcal{F}_{t}, P\right)$. Alaton et al. [1] consider the same dynamics, except that the volatlity $\sigma(t)$ is assumed to be constant for each month, whereas Dornier and Querel [17] assume a constant volatility in their seminal paper. The dynamics (14) is generalized to a so-called continuous-time AR (CAR) process in Benth et al. [7], which is applied to Stockholm data. Later, Härdle and Lopez Cabrera [19] studied this class of processes for German temperature data, and Benth et al. [9] for Asian temperatures, all validating the relevance of this class of models. When estimating these models, discretization of the stochastic process is applied leading back to the time series models discussed and analysed in the 

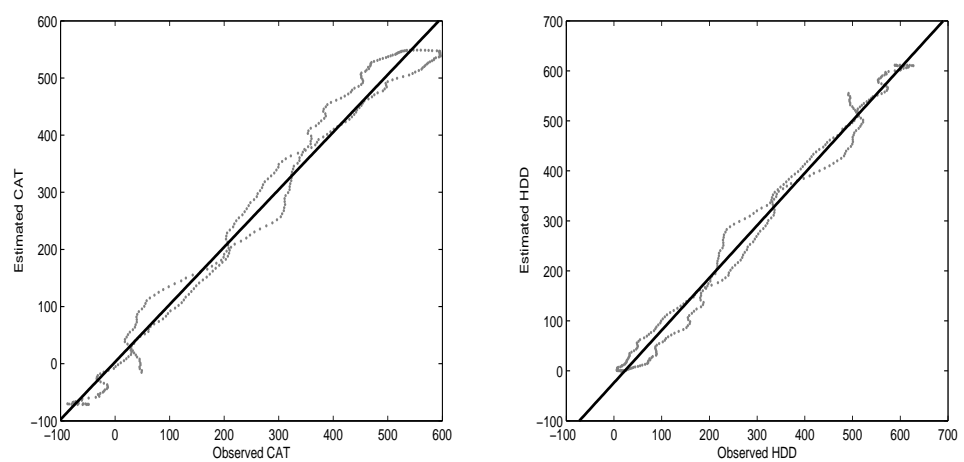

Figure 10: Scatter plot of observed vs. estimated CAT (left) and HDD (right) indices for year 2004 calculated from a 30 days window.

present paper.

Temperatures are naturally evolving continuously over time, so it is very appealing to use continuous-time stochastic processes to model the dynamics although the data may be on a daily scale and the weather derivatives market settles contracts based on indices of daily average temperature. There is another fundamenatal aspect related to the nature of the temperature futures markets. Temperature futures can be traded continuously in the opening hours of the exchange. Thus, a model for the forward price dynamics is naturally formulated as a continuous-time stochastic process.

A standard definition (see Benth et al [8]) for the dynamics of temperature futures prices is to use the risk-adjusted predicted index value, given today's information modelled by the filtration $\mathcal{F}_{t}$,

$$
F_{\mathrm{I}}\left(t, T_{1}, T_{2}\right)=\mathbb{E}_{Q}\left[\mathrm{I}\left(T_{1}, T_{2}\right) \mid \mathcal{F}_{t}\right]
$$

with I being one of the indices CAT, HDD or CDD, and measurement period $\left[T_{1}, T_{2}\right]$. To take into account a compensation for risk bearing, the expectation 
is computed under a pricing probability $Q$, denoted $\mathbb{E}_{Q}$. To compute prices, we need to know the probabilistic properties of the index $\mathrm{I}\left(T_{1}, T_{2}\right)$ under $Q$, that is, of the temperature dynamics under $Q$. In continuous-time models of Brownian motion type, this can be achieved by a Girsanov transform which effectively shifts the seasonal function by some constant usually called the market price of risk (we refer the reader to Benth et al. [8] for details on this topic). Using the "burn-in" approach of Jewson and Brix [21] is in some sense corresponding to choosing $Q=P$, that is, no risk premium is introduced in the forward prices. We note that the seasonal mean function will significantly influence the futures price dynamics as can be seen from (15). This again argues for the importance of having an accurate description of the seasonal mean $S(t)$.

With the continuous-time models for temperatures proposed in (14), or more general CAR models, we can easily compute the price dynamics of CAT, CDD and HDD futures (see Benth et al. [8] for details). With a time series approach, as suggested by Campbell and Diebold [14], this becomes a cumbersome task. Firstly, we will obtain a time series model for the futures price dynamics, with time measured on a daily scale. This could of course be easily mended by defining the model on a finer time scale. But more importantly, the dynamics is rather complex and one needs to resort to simulations in order to calculate the conditional expectations in (15). An alternative to this is to try to reformulate the Campbell and Diebold model as a continuous-time stochastic process.

Since the weather market at CME offers trade in European options on temperature futures, it is of big value to have an explicit dynamics of the 
futures prices. In fact, from Benth et al. [8], we can derive analytic prices for options on CAT futures. With the HDD and CDD futures dynamics one cannot obtain explicit option prices, however, they will be reasonably simple to simulate. If we use a time series model for temperatures of some complexity, option prices must be simulated from scenarios of futures prices, involving a computer-intensive nested simulation procedure. The question of hedging is also easily treated in a continuous-time framework (see Benth et al. [8]), in contrast to a time series approach.

We have proposed a model for the residuals $\varepsilon(t)$ based on a multiplicative structure of variance seasonality and GARCH effects. There are many continuous-time stochastic volatility models which share common properties with the GARCH dynamics in discrete time, and we here briefly discuss the Barndorff-Nielsen and Shephard model (BNS) introduced in [2]. Suppose that the deseasonalized temperature dynamics is given by

$$
d \widetilde{Z}(t)=-\alpha \widetilde{Z}(t) d t+\sigma_{\mathrm{BSB}}(t) \sqrt{V(t)} d B(t),
$$

with

$$
d V(t)=-\lambda V(t) d t+d L(\lambda t)
$$

Here, $\lambda>0$ is a constant measuring the speed of mean reversion for the volatility process $V(t)$, which reverts to zero. The process $L(t)$ is assumed to be a subordinator independent of $B$, the Brownian motion, meaning a Lévy process with increasing paths. In this way one is ensured that $V(t)$ is positive. The unusual time scaling by $\lambda$ in the argument of $L$ is convenient when estimating such a stochastic volatlity model. In fact, one may get relatively explicit distributions for the "deseasonalized" residuals $\sqrt{Z(t)} d B(t)$, which 
become conditionally normal, with mean zero and variance $V(t)$. In stationarity of $V$, this distribution becomes independent of $\lambda$, and therefore one may separate the modelling of these residuals from the dependency structure in the paths. For this stochastic volatility model, the squared residuals will have an exponentially decaying ACF, with decay rate $\lambda$. By subordination of such $V$ 's, the ACF may decay as a sum of exponentials. We refer to Barndorff-Nielsen and Shephard [2] for an extensive analysis of this class of stochastic volatility models. We remark that this stochastic volatility model is easily included in $\operatorname{CAR}(p)$ processes, see Benth and Šaltytė Benth [6]. We emphasize that the stochastic BNS volatility does not become a GARCH dynamics in discrete time, but is applied here as an alternative sharing some simular properties as GARCH.

\section{References}

[1] Alaton, P., Djehiche, B. and Stillberger, D. (2002). On modelling and pricing weather derivatives. Appl. Math. Finance, 9(1), 1-20.

[2] Barndorff-Nielsen, O. E., and Shephard, N. (2001). Non-Gaussian Ornstein-Uhlenbeck-based models and some of their uses in economics. J. R. Statist. Soc., B, 63(2), 167-241 (with discussion).

[3] Benth, F. E. and Saltyte-Benth, J. (2004). The normal inverse Gaussian distribution and spot price modelling in energy markets. Intern. J. Theor. Appl. Finance, 7(2), 177-192. 
[4] Benth, F. E. and Šaltytė-Benth, J. (2005). Stochastic modelling of temperature variations with a view towards weather derivatives. Appl. Math. Finance, 12(1), 53-85.

[5] Benth, F. E. and Šaltytė-Benth, J. (2007). The volatility of temperature and pricing of weather derivatives. Quant. Finance, 7(5), 553-561.

[6] Benth, F. E. and Šaltytė Benth, J. (2010). Weather derivatives and stochastic modelling of temperature. Manuscript, University of Oslo.

[7] Benth, F. E., Šaltytè-Benth, J. and Koekebakker, S. (2007). Putting a price on temperature. Scand. J. Statist, 34, 746-767.

[8] Benth, F. E., Šaltytė-Benth, J. and Koekebakker, S. (2008). Stochastic Modelling of Electricity and Related Markets. World Scientific.

[9] Benth, F. E., Härdle, W. and Lopez-Cabrera, B. (2009). Pricing of Asian temperature risk. SFB 649 Discussion Paper 2009-046, Humboldt Universitat zu Berlin.

[10] Bollerslev, T. (1986). Generalized autoregressive conditional heteroscedasticity. J. Econometrics, 31, 307-327.

[11] Brody, D. C., Syroka, J. and Zervos, M. (2002). Dynamical pricing of weather derivatives. Quantit. Finance, 3, 189-198.

[12] Cao, M., and Wei, J. (2004). Weather derivatives valuation and market price of weather risk. J. Futures Markets, 24, 1065-1089.

[13] Campbell, S. D. and Diebold, F. X. (2002). Weather forecasting for weather derivatives. Manuscript. Available on 
http://www.ssc.upenn.edu/ fdiebold/papers/papers.html. Version: December 4, 2002.

[14] Campbell S.D. and Diebold F.X. (2005). Weather forecasting for weather derivatives. J. Amer. Statist. Assoc., 100 (469), pp. 6-16.

[15] Chao, Ch.-Ch., Lin, J.-B., and Shen, W.-M. (2009). Pricing weather derivatives using a predictive power time series process. Asia-Pacific J. Fin. Studies, 38 (6), pp. 863-890.

[16] Davis, M. H. A. (2001). Pricing weather derivatives by marginal value. Quantit. Finance, 1, 305-308.

[17] Dornier, F. and Querel, M. (2000). Caution to the wind. Energy Power Risk Manag., Weather risk special report, August, 30-32.

[18] Folland, G. (1984). Real Analysis. John Wiley \& Sons.

[19] Härdle, W., and Lopez Cabrera, B. (2009). Infering the market price of weather risk. Discussion paper, SFB 649, Humboldt-Universitt zu Berlin.

[20] Härdle, W., Lopez Cabrera, B., and Wang, W. (2010). Localizing temperature residuals. Manuscript in preparation.

[21] Jewson, S., and Brix, A. (2005). Weather Derivative Valuation. Cambridge University Press.

[22] Karatzas, I., and Shreve, S. E. (1991). Brownian Motion and Stochastic Calculus, Springer-Verlag, New York. 
[23] Mraoua, M. and Bari, D. (2007). Temperature stochastic modeling and weather derivatives pricing: empirical study with Maroccan data. Afrika Statistika, 2, 22-43.

[24] Papazian, G. and Skiadopoulos, G.S. (2010). Modeling the dynamics of temperature with a view to weather derivatives. Available at SSRN: http://ssrn.com/abstract=1517293. Version: January 19, 2010.

[25] Šaltytė Benth J., Benth, F. E. and Jalinskas P. (2007). A spatialtemporal model for temperature with seasonal variance. J. Appl. Statist., 34, 823-841.

[26] Schiller, F., Seidler, G., and Wimmer, M. (2010). Temperature models for pricing weather derivatives. Quantit. Finance, Forthcomming. Available at SSRN: http://ssrn.com/abstract=1280826. Version: March 11, 2010.

[27] Svec, J. and Stevenson, M. (2007). Modelling and forecasting temperature based weather derivatives. Global Finance J., 18, 185-204. 